EM-DAT database, floods account for $38.7 \%$ of all incidences, $6.2 \%$ of the deaths and every year $43 \%$ of world population is affected by flood. There are various factors which magnify the impact of flood to the community in the catchment area. The nature and extent of the flood is determined by the physical location and topography, and by the built environment. The built environment comprises of the existing political, social and economic structures inherent in a community. In the post-flood period, the risk of diarrhoea is significantly higher for those with lower educational level, living in a household with a non-concrete roof, drinking tube-well water (vs. tap water), using a distant water source and unsanitary toilets. The 1998 Bangladesh flood study confirms that low socio-economic groups and poor hygiene and sanitation groups are most vulnerable to flood-related diarrhoea (Annya et al., 2010). The Bihar flood of 2008 affected 100 villages out of which Supaul, Araria, Saharsa, Madhepura and Purnea were the most severly affected districts. Nearly 2.5 million people were affected by the floodwater. Various disease outbreaks are a common phenomenon in a post flood scenario. In Bihar, the population witnessed outbreaks of malaria, diarrhoea, measles and cholera. This paper examines the various factors causing diarrhoeal spread in the five flood affected districts of Bihar. Also, the paper analyses the above factors in terms of most contributing and least contributing factor towards diarrhoeal outbreak. The methodology used for the study is the statistical tool of Standard Multiple Regression Analyses where several independent variables will predict the dependent variable. The various independent variables have been taken all the five affected districts in Bihar floods. They are malnutrition, population density, awareness levels, socio-economic conditions, literacy, extent of flood and availability of health facilities. These independent variables will determine how much each predicts the dependent variable and as a result help in analysing the linear relationship between the dependent and independent variables.

Prehosp Disaster Med 2011;26(Suppl. 1):s68-s69 doi:10.1017/S1049023X11002354

(A252) Impact of Protracted, Intrastate Conflict on Population Health in Manipur, India V. Kaushik, ${ }^{1}$ S. Nair ${ }^{1}$ Y. Tanwar, ${ }^{1}$ S. Sinha,${ }^{1}$ N. Roy ${ }^{2}$

1. Tata Institute of Social Sciences, Mumbai, India

2. Public Health, Mumbai, India

Introduction: Manipur is a state in northeastern India and in civil war for $>45$ years. Healthcare delivery and access is affected due to poor security, restricted accessibility, and the incapacity of this fragile state.

Methods: The burden of morbidity and mortality in the conflict area of Manipur was estimated using data sources (hospital attendance, hospital inpatient, and death registries, national family and health registries, and in-depth interviews of healthcare providers) and compared to national averages. These findings were co-related with violent events reported in the local newspaper.

Results: Excess mortality was observed in the $21-50$ year age group, but not in females or the elderly. The major causes of deaths were non-communicable diseases, cerebrovascular accidents, and chronic pulmonary disease. Chronic conflict increased the burden of alcohol liver disease and of mental health diseases. Suicidal deaths were common in the midtwenty age group and usually due to agricultural pesticide consumption. These deaths were higher in men, and suicide attempts were higher in women. The prevalence of intravenous drug users and of HIV was reported to be five times as higher than the national average. High rates of disappearances, mutilation, torture, kidnapping, and hostage-taking, spousal physical violence and attacks on healthcare facilities and medical personnel were events of concern. There were no reported events of suicide bombers.

Conclusions: Protracted conflict dramatically changes the demographics and disease burden. Humanitarian space constantly is under threat of attack and the insecurity interferes with the provision of sustained preventive and curative services. Recommendations to be implemented would measures to continue treatment in the insecure environment through telephonic or online medical helplines, vaccination, and drug supplies during negotiated ceasefires or curfew times and protecting humanitarian spaces. However, militarization of healthcare may not be favorable solution.

Prehosp Disaster Med 2011;26(Suppl. 1):s69

doi:10.1017/S1049023X11002366

\section{(A253) Integrated Community-Based Interventions to Overcome a Deadly Cholera Outbreak in Zimbabwe M.N. Yao}

Health Action in Crisis, Geneva, Switzerland

An unprecedented cholera outbreak affected Zimbabwe from August 2008 to July 2009 with 98,592 cases and 4,288 deaths, in 54 out of 62 districts. The main strategy used to overcome the outbreak was an integrated community-based interventions package. The present work is a case study to describe the strategy and lessons learned for future humanitarian crises and preparedness. The methodology was based on the review of epidemiological reports, assessment and surveys' reports, minutes of joint Health and Water Sanitation and Hygiene (WASH) Clusters' meetings, and direct observation as Health Cluster Coordinator. Epidemiological data showed an increasing number of cases in rural areas with community deaths representing $66 \%$ of the 1,948 deaths from 61,304 cases on 31 January 2009. Risk factors identified in communities were: lack of awareness about the disease, cultural and religious behaviors, lack of potable water with weak sanitation, lack and inappropriate use of water purification tablets, and lack of soap and water containers for effective behavior change. There also was late arrival to cholera to the few treatment centers by rural populations. In addition to treatment centers, a package of interventions was implemented by multi-sectoral stakeholders. The package included: health and WASH education tools and practice sessions for healthy and hygienic behavior change and for an effective use of oral rehydration salt as first aid measure; community-based surveillance with an early warning system and response teams; and distribution of containers and water purification tablets with drilling of water points. Epidemiological data showed a significant decrease of cholera cases where the full package was implemented. This work showed that an integrated package of interventions jointly targeting risk factors 